\title{
ARO Biological Models Workshop Auditory Neurophysiology: Relevance of Animal Studies to Human Perception
}

\section{Introduction}

\author{
Bryan E. Pfingst \\ Kresge Hearing Research Institute, Department of Otorhinolaryngology, \\ University of Michigan, U.S.A.
}

The five papers which follow are the result of a workshop held in February, 1987 at the Tenth Midwinter Meeting of the Association for Research in Otolaryngology, organized at the request of the then ARO president, Donald $W$. Nielsen.

The focus of the workshop was evaluation of an animal model for studies applicable to humans. For this purpose we chose one of the most prevalent, timely, and imperative of animal models used in Otolaryngology: the mammalian single-unit preparation for studies of encoding and processing of acoustic information by the auditory nervous system. Because of time constraints, we focused on the use of this model for studies of encoding frequency and level information in simple and complex signals.

We chose three approaches to selecting and evaluating the animal model. The first was anatomically based. By comparing structural similarities and differences across species onc can makc informed guesses about functional similarities. The second approach was to compare predictions of neural models derived from the single-unit data with known human psychoacoustic data to assess the validity of the neural models. Finally, a comparative psychoacoustics approach was used to establish the limits of generalizing from the animal model to humans.

Our goal here was not to accept or reject this approach to studying the nervous system. The fact is that we are dependent on this approach unless a noninvasive way of studying single-unit activity is found. In evaluating how accurately the animal model approximates the human condition, we hope to accomplish two things. In cases where the animal model accurately reflects human anatomy, physiology, and behavior, we feel it appropriate to make direct applications to human problems. In cases where obvious differences exist, a comparative approach may help elucidate mechanisms of perception. Thus the goal of this exercise was to understand more clearly how we can most intelligently apply the results obtained from studies using the animal model.

The workshop was organized in three sets of two papers each. The comparative CNS anatomy paper by Jean K. Moore is represented in a previous publication in Hearing Research $(1987,29,1-31)$ and will not be reproduced here.

My thanks to Ben M. Clopton, Joseph E. Hawkins, and Gregory H. Wakefield for their help in conducting the workshop and to many anonymous reviewers for their insightful comments on the manuscripts.

Editors Note:

All papers were reviewed in the same way as regular contributions to Hearing Research.

Aage R. Meller, Ph.D. 